\title{
CORRELATIONS OF THE ARRIVAL DIRECTIONS OF ULTRA-HIGH ENERGY COSMIC RAYS WITH EXTRAGALACTIC OBJECTS AS OBSERVED BY THE TELESCOPE ARRAY EXPERIMENT
}

\author{
T. Abu-Zayyad ${ }^{1}$, R. Aida ${ }^{2}$, M. Allen ${ }^{1}$, R. Anderson ${ }^{1}$, R. Azuma $^{3}$, E. Barcikowski ${ }^{1}$, J. W. Belz $^{1}$, D. R. Bergman ${ }^{1}$, \\ S. A. Blake ${ }^{1}$, R. Cady ${ }^{1}$, B. G. CheOn $^{4}$, J. Chiba ${ }^{5}$, M. Chikawa ${ }^{6}$, E. J. Cho ${ }^{4}$, W. R. Cho ${ }^{7}$, H. Fujir ${ }^{8}$, T. Fujir ${ }^{9}$, T. Fukuda ${ }^{3}$, \\ M. Fukushima ${ }^{10,11}$, W. Hanlon ${ }^{1}$, K. Hayashi ${ }^{3}$, Y. Hayashi ${ }^{9}$, N. Hayashida ${ }^{10}$, K. Hibino ${ }^{12}$, K. HiYama ${ }^{10}$, K. Honda $^{2}$,

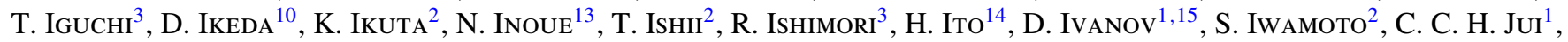 \\ K. Kadota ${ }^{16}$, F. KaKimoto ${ }^{3}$, O. Kalashev ${ }^{17}$, T. Kanbe ${ }^{2}$, K. Kasahara ${ }^{18}$, H. Kawai ${ }^{19}$, S. KawaKami ${ }^{9}$, S. Kawana ${ }^{13}$,

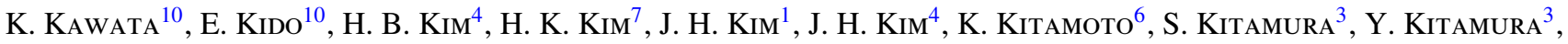 \\ K. Kobayashi ${ }^{5}$, Y. Kobayashi ${ }^{3}$, Y. Kondo ${ }^{10}$, K. Kuramoto ${ }^{9}$, V. KuZmin ${ }^{17}$, Y. J. Kwon ${ }^{7}$, J. LAN ${ }^{1}$, S. I. Lim ${ }^{20}$, J. P. Lundquist ${ }^{1}$, \\ S. Machida $^{3}$, K. Martens ${ }^{11}$, T. Matsuda ${ }^{8}$, T. Matsuura ${ }^{3}$, T. Matsuyama ${ }^{9}$, J. N. Matthews ${ }^{1}$, M. Minamino ${ }^{9}$, K. Miyata $^{5}$,

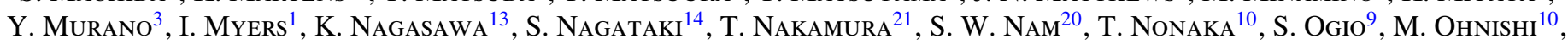

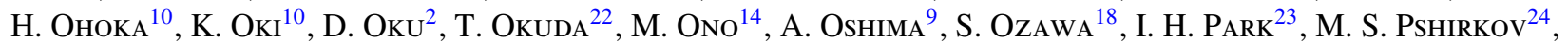 \\ D. C. Rodriguez ${ }^{1}$, S. Y. Roh ${ }^{25}$, G. Rubtsov ${ }^{17}$, D. Ryu ${ }^{25}$, H. SAgawa ${ }^{10}$, N. SAKurai ${ }^{9}$, A. L. SAMPSON ${ }^{1}$, L. M. Scott ${ }^{15}$, \\ P. D. Shah ${ }^{1}$, F. Shibata ${ }^{2}$, T. Shibata ${ }^{10}$, H. Shimodaira ${ }^{10}$, B. K. Shin ${ }^{4}$, J. I. Shin ${ }^{7}$, T. Shirahama ${ }^{13}$, J. D. Smith ${ }^{1}$, \\ P. Sokolsky ${ }^{1}$, R. W. Springer ${ }^{1}$, B. T. Stokes ${ }^{1}$, S. R. Stratton ${ }^{1,15}$, T. Stroman ${ }^{1}$, S. Suzuki ${ }^{8}$, Y. TAKahashi ${ }^{10}$, M. Takeda $^{10}$,

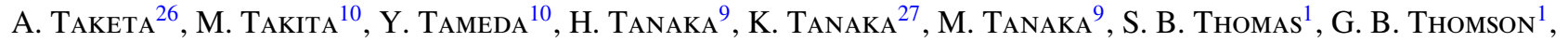

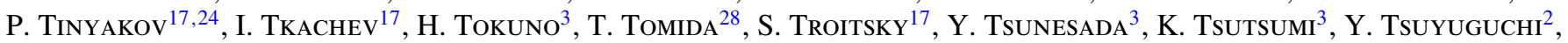 \\ Y. UChIHORI ${ }^{29}$, S. Udo ${ }^{12}$, H. UKAI ${ }^{2}$, F. URBAN ${ }^{24}$, G. VASILOFF ${ }^{1}$, Y. WAdA ${ }^{13}$, T. WONG ${ }^{1}$, Y. YAMAKAWA ${ }^{10}$, R. YAMANE ${ }^{9}$,

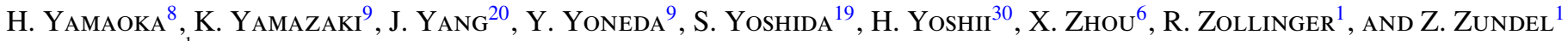 \\ ${ }^{1}$ High Energy Astrophysics Institute and Department of Physics and Astronomy, University of Utah, Salt Lake City, UT, USA \\ ${ }^{2}$ Interdisciplinary Graduate School of Medicine and Engineering, University of Yamanashi, Kofu, Yamanashi, Japan \\ ${ }^{3}$ Graduate School of Science and Engineering, Tokyo Institute of Technology, Meguro, Tokyo, Japan \\ ${ }^{4}$ Department of Physics and The Research Institute of Natural Science, Hanyang University, Seongdong-gu, Seoul, Korea \\ ${ }^{5}$ Department of Physics, Tokyo University of Science, Noda, Chiba, Japan \\ ${ }^{6}$ Department of Physics, Kinki University, Higashi Osaka, Osaka, Japan \\ ${ }^{7}$ Department of Physics, Yonsei University, Seodaemun-gu, Seoul, Korea \\ ${ }^{8}$ Institute of Particle and Nuclear Studies, KEK, Tsukuba, Ibaraki, Japan \\ ${ }^{9}$ Graduate School of Science, Osaka City University, Osaka, Japan \\ ${ }^{10}$ Institute for Cosmic Ray Research, University of Tokyo, Kashiwa, Chiba, Japan \\ ${ }^{11}$ Kavli Institute for the Physics and Mathematics of the Universe (WPI), Todai Institutes for Advanced Study, \\ the University of Tokyo, Kashiwa, Chiba, Japan \\ ${ }^{12}$ Faculty of Engineering, Kanagawa University, Yokohama, Kanagawa, Japan \\ 13 The Graduate School of Science and Engineering, Saitama University, Saitama, Japan \\ ${ }^{14}$ Astrophysical Big Bang Laboratory, RIKEN, Wako, Saitama, Japan \\ ${ }^{15}$ Department of Physics and Astronomy, Rutgers University, Piscataway, NJ, USA \\ ${ }^{16}$ Department of Physics, Tokyo City University, Setagaya-ku, Tokyo, Japan \\ ${ }^{17}$ Institute for Nuclear Research of the Russian Academy of Sciences, Moscow, Russia \\ ${ }^{18}$ Advanced Research Institute for Science and Engineering, Waseda University, Shinjuku-ku, Tokyo, Japan \\ ${ }^{19}$ Department of Physics, Chiba University, Chiba, Japan \\ ${ }^{20}$ Department of Physics and Institute for the Early Universe, Ewha Womans University, Seodaaemun-gu, Seoul, Korea \\ ${ }^{21}$ Faculty of Science, Kochi University, Kochi, Japan \\ ${ }^{22}$ Department of Physical Sciences, Ritsumeikan University, Kusatsu, Shiga, Japan \\ ${ }^{23}$ Sungkyunkwan University, Jang-an-gu, Suwon, Korea \\ ${ }^{24}$ Service de Physique Théorique, Université Libre de Bruxelles, Brussels, Belgium \\ ${ }^{25}$ Department of Astronomy and Space Science, Chungnam National University, Yuseong-gu, Daejeon, Korea \\ ${ }^{26}$ Earthquake Research Institute, University of Tokyo, Bunkyo-ku, Tokyo, Japan \\ ${ }^{27}$ Department of Physics, Hiroshima City University, Hiroshima, Japan \\ ${ }^{28}$ Advanced Science Institute, RIKEN, Wako, Saitama, Japan \\ ${ }^{29}$ National Institute of Radiological Science, Chiba, Chiba, Japan \\ ${ }^{30}$ Department of Physics, Ehime University, Matsuyama, Ehime, Japan \\ Received 2013 June 15; accepted 2013 September 2; published 2013 October 17
}

\begin{abstract}
We search for correlations between the positions of extragalactic objects and the arrival directions of ultra-high energy cosmic rays (UHECRs) with primary energy $E \geqslant 40 \mathrm{EeV}$ as observed by the surface detector array of the Telescope Array (TA) experiment during the first 40 months of operation. We examine several public astronomical object catalogs, including the Veron-Cetty and Veron catalog of active galactic nuclei. We count the number of TA events correlated with objects in each catalog as a function of three parameters: the maximum angular separation between a TA event and an object, the minimum energy of the events, and the maximum redshift of the objects. We determine the combination of these parameters that maximizes the correlations, and we calculate the probability of
\end{abstract}


having the same levels of correlations from an isotropic distribution of UHECR arrival directions. No statistically significant correlations are found when penalties for scanning over the above parameters and for searching in several catalogs are taken into account.

Key words: acceleration of particles - astroparticle physics - cosmic rays

Online-only material: color figures

\section{INTRODUCTION}

The origin of ultra-high energy cosmic rays (UHECRs) is one of the most important unsolved mysteries in modern astrophysics (e.g., Kotera \& Olinto 2011). It is generally thought that cosmic rays with energies greater than $10^{18} \mathrm{eV}(1 \mathrm{EeV})$ are of extragalactic origin because the Galactic magnetic fields are not strong enough to confine them. Indeed, no apparent anisotropy in the arrival directions of UHECRs along the Galactic plane has been found. On the other hand, a steepening in the energy spectrum of UHECRs at around $50 \mathrm{EeV}$ is observed by the High Resolution Fly's Eye (HiRes) experiment and the Telescope Array (TA) experiment (Abbasi et al. 2008b; Abu-Zayyad et al. 2013), as well as by the Pierre Auger Observatory in a similar energy region (Abraham et al. 2008, 2010). This can be explained as a consequence of the cosmic ray energy losses due to interactions with the cosmic microwave background, as predicted by Greisen (1966) and Zatsepin \& Kuz'min (1966, GZK).

In this case, we expect that most of the observed cosmic rays of the highest energies originate from sources within the GZK horizon $(\sim 100 \mathrm{Mpc})$, and a correlation between nearby objects and arrival directions of cosmic rays is expected. The UHECRs are deflected by the Galactic and extragalactic magnetic fields on their way to Earth. The deflection angles are determined by the particle charges, source distances, and strength of the magnetic fields. For example, in the case of a proton arriving from a $100 \mathrm{Mpc}$ distance through a random extragalactic magnetic field with a strength of $1 \mathrm{nG}$ and a correlation length of $\sim 1 \mathrm{Mpc}$, the expected deflection angle is $3^{\circ}-5^{\circ}$ for $100 \mathrm{EeV}$ (and less than $15^{\circ}$ for $40 \mathrm{EeV}$ ) when using the existing magnetic field estimates (Han et al. 2006; Sun et al. 2008; Pshirkov et al. 2011; Kronberg 1994).

The TA experiment observes UHECRs in the northern hemisphere by using a surface detector (SD) array (Abu-Zayyad et al. $2012 \mathrm{~b}$ ) of $\sim 700 \mathrm{~km}^{2}$ area located in Millard County, Utah, USA (39.3 N, 112.9 W). Three fluorescence detector (FD) stations (Tokuno et al. 2012; Abu-Zayyad et al. 2012c) surround the SD array (Kawai et al. 2008) and view the atmosphere above it. The SD array consists of 507 SDs installed on a square grid with $1.2 \mathrm{~km}$ spacing and measures particles from extensive air showers (EASs) at ground level. The energy and the arrival direction of a primary particle are determined from observed energy deposits as a function of distance from the shower core in the SDs and the arrival time distribution of the EAS particles. The test operation of the SD array began in 2008 March, and the full SD array has been operational with uniform trigger criteria since 2008 May 11. The present analysis uses only the events detected by the SD array because this data set has a larger statistical sample than the data set from the FDs.

Assuming the sources have the same intrinsic UHECR luminosities, the arrival directions of higher energy cosmic rays from nearby sources are expected to correlate better with the source positions. We search for the correlations between the TA events and objects in catalogs by changing three parameters: the minimum energy of the cosmic ray events, $E_{\min }$, the separation angle, $\psi$, between the cosmic ray arrival direction and the object, and the maximum redshift, $z_{\max }$, of the objects. A similar approach has been taken in the analyses by the Pierre Auger Observatory (Abreu et al. 2007, 2008, 2010) and by the HiRes experiment (Abbasi et al. 2008a) using the Veron-Cetty and Veron (VCV) catalog of 12 th edition (Veron-Cetty \& Veron 2006).

We examine the objects in the 13th edition of the VCV catalog (Veron-Cetty \& Veron 2010) as putative sources of UHECRs. This catalog is a compilation of several surveys made under different conditions such as field of views (FOVs), observation periods, etc. It does not represent a homogeneous sample of active galactic nuclei (AGNs), and its degree of completeness is unknown (Veron-Cetty \& Veron 2010). In addition, we have investigated unbiased data sets from different measurements, namely, radio: the third Cambridge catalog of radio sources catalog (3CRR; Laing et al. 1983); infrared: the 2MASS (the Two Micron All Sky Survey) redshift survey catalog (2MRS; Huchra et al. 2012); X-ray: the Swift BAT (Burst Alert Telescope) 58 month hard X-ray survey catalog (SB-58M; Tueller et al. 2010), and the 60 month AGN survey catalog (SB-AGN; Ajello et al. 2012); and gamma ray the 2nd Fermi Large Area Telescope (LAT) AGN catalog (2LAC; Ackermann et al. 2011). In each catalog, we select only those objects that have redshift information. In the case of the $2 \mathrm{LAC}$ catalog, this criterion reduces the number of objects by $\sim 50 \%$.

The paper is organized as follows. The observation status of the SD array and the qualities of reconstructed events are briefly described in Section 2. The details of the parameter scanning in the correlation searches using the object catalogs are given in Section 3, and the results are described in Section 4. We also investigated penalties for the multi-catalog scanning (PCSs) in Section 5. The conclusions from this analysis are in Section 6.

\section{SD DATA}

In this work we use the SD air shower events observed in the first 40 month run of TA from 2008 May to 2011 September. These events are triggered by a three-fold coincidence of adjacent SDs within $8 \mu$ s (Abu-Zayyad et al. 2012b).

The details of the SD event reconstruction are described elsewhere (Ivanov et al. 2011; Abu-Zayyad et al. 2013). First, the shower geometry including the arrival direction is obtained by using the time differences between the observed signals at each SD. Next, the precise shower geometry and the lateral distribution of shower particles are determined using the observed energy deposit in each SD. Finally, the primary energy is determined from the lateral distribution. The overall energy scale of the SD events is fixed by calibration with the FD energy scale using a hybrid event set as described by Abu-Zayyad et al. (2011). The systematic uncertainty in energy determination is $22 \%$.

The data quality cuts for the reconstructed events are the same as in the previous TA analysis papers (Abu-Zayyad et al. 2012a, 2013). The events are cut if the zenith angle is greater 

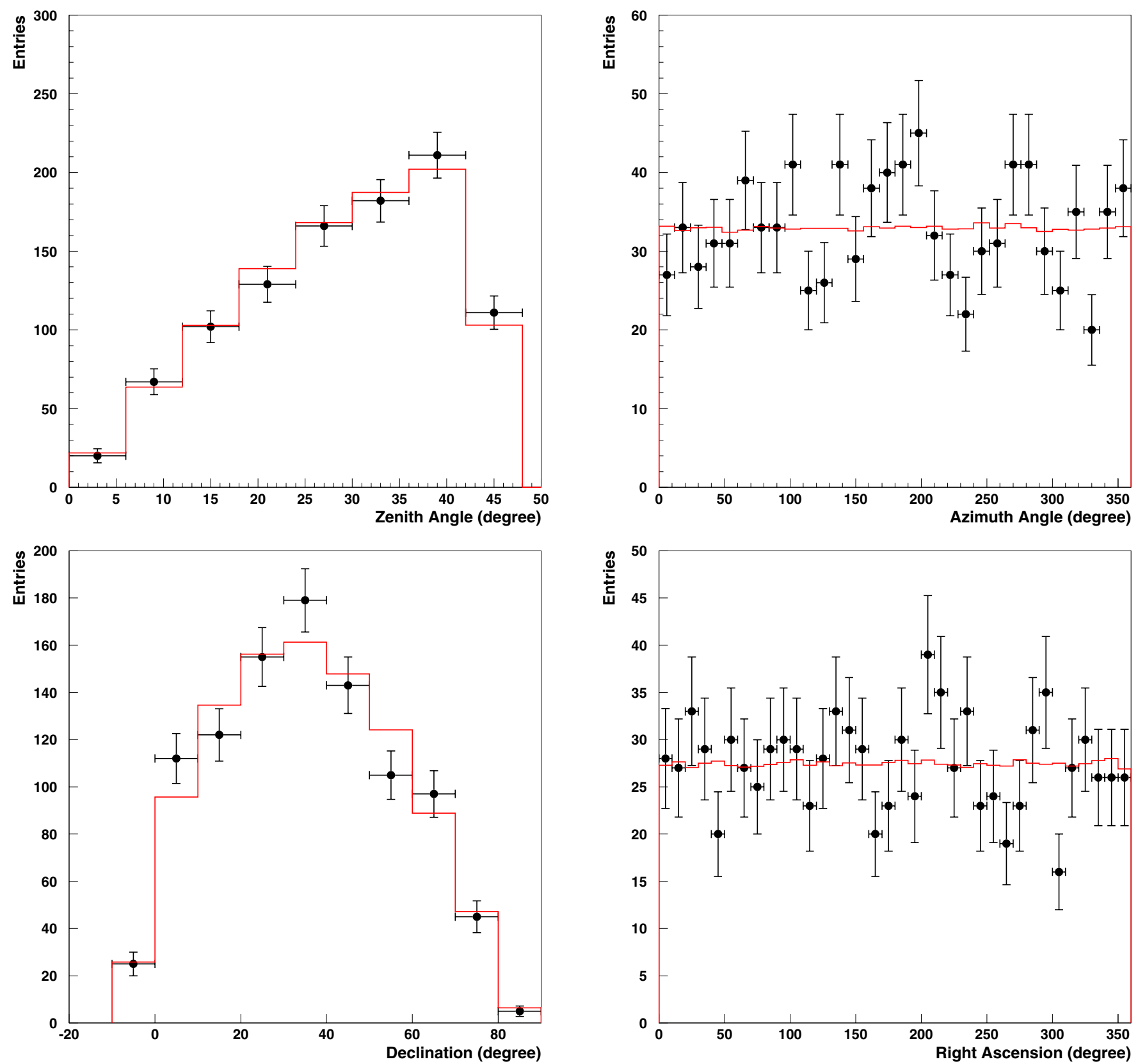

Figure 1. Distribution of the observed data (plot) and the simulated data with the geometrical acceptance (histogram) with energy $>10$ EeV. Top left: zenith angle; top right: azimuth angle; bottom left: declination; and bottom right: right ascension.

(A color version of this figure is available in the online journal.)

than $45^{\circ}$ and/or the core position is within $1200 \mathrm{~m}$ of the SD array boundary. The EAS reconstruction efficiency under these criteria is greater than $98 \%$, including the duty cycle of the SD array for $E>10 \mathrm{EeV}$ (Abu-Zayyad et al. 2012b, 2013). The accuracy of arrival direction determination is 1.5 , and the energy resolution is better than $20 \%$ in this energy range.

The number of events remaining after reconstruction and quality cuts is 988 for $E \geqslant 10 \mathrm{EeV}, 57$ for $E \geqslant 40 \mathrm{EeV}$, and 3 for $E \geqslant 100 \mathrm{EeV}$. From our Monte Carlo studies including the full detector response simulations, we confirm that the acceptance of the SD array is fully geometrical, i.e., independent of the arrival direction up to $\theta=45^{\circ}$ for showers with energies greater than $10 \mathrm{EeV}$ (Abu-Zayyad et al. 2012a, 2012b, 2013). We also confirm that the arrival direction distribution of the observed events in the horizontal coordinates and the equatorial coordinates are consistent with large-scale isotropy shown in Figure 1. In this analysis, we use the geometrical acceptance to generate random events for reasons of computational efficiency. The total exposure of the SD array in the first 40 months of operation is $3.1 \times 10^{3} \mathrm{~km}^{2} \mathrm{sr} \mathrm{yr}$, including the quality cuts.

\section{CORRELATION ANALYSIS}

\subsection{Object Catalogs}

We use the catalogs of extragalactic objects that are derived from measurements as listed in Table 1. In several catalogs, the objects near the Galactic plane are excluded to avoid incompleteness from the experimental limitation by the authors of each catalog. We also exclude the observed SD events in the corresponding regions. 
Table 1

Configurations of the Used Catalogs

\begin{tabular}{llrr}
\hline \hline Catalog & \multicolumn{1}{c}{ Range } & \multicolumn{1}{c}{$N_{\text {all }}$} & $N_{\text {target }}$ \\
\hline 3CRR & Compilation of radio surveys & 173 & 16 \\
2MRS & IR $(1-2 \mu \mathrm{m})$ & 43533 & 13547 \\
SB-58M & X-ray $(14-195 \mathrm{keV})$ & 1092 & 161 \\
SB-AGN & X-ray $(15-55 \mathrm{keV})$ & 428 & 102 \\
2LAC & $\gamma$-ray $(100 \mathrm{MeV}-100 \mathrm{GeV})$ & 1126 & 6 \\
VCV & Compilation of AGNs & 168941 & 762 \\
\hline
\end{tabular}

Note. $N_{\text {all }}$ : number of all objects contained within the catalog, $N_{\text {target }}$ : number of objects with the redshift $z<0.03$ within the TA FOV.

Table 2

Regions Cut Away from the Galactic Plane of Each of the Catalogs and the Number $(N)$ of Events Remaining (the Maximum Number is 57)

\begin{tabular}{llc}
\hline \hline Catalog & \multicolumn{1}{c}{ Cut Region $(\mathrm{deg})$} & $N(E \geqslant 40 \mathrm{EeV})$ \\
\hline 3 CRR & $|b|<10^{\circ}, \delta<10^{\circ}$ & 41 \\
2MRS & $|b|<5^{\circ}$ for $30^{\circ} \leqslant l \leqslant 330^{\circ}$ & 56 \\
& $|b|<8^{\circ}$ otherwise & \\
SB-58M & None & 57 \\
SB-AGN & None & 57 \\
2LAC & $|b|<10^{\circ}$ & 49 \\
VCV & None & 57 \\
\hline
\end{tabular}

Note. $b$ : Galactic latitude, $l$ : Galactic longitude, and $\delta$ : declination of the equatorial coordinate.

The target objects and the cut criteria in each of the catalogs are summarized below. These criteria (e.g., significance level) were chosen by the authors of each catalog. The 3CRR catalog contains radio galaxies detected at $178 \mathrm{MHz}$ with fluxes greater than 10 Jy (Laing et al. 1983). Objects in the direction of the Galactic disk $\left(|b|<10^{\circ}\right.$ ) were not included. The 2MRS (Huchra et al. 2012) catalog is derived from the 2MASS observation with a detection range between $1-2 \mu \mathrm{m}$ and $K_{\mathrm{s}} \leqslant 11.75 \mathrm{mag}$. This catalog also loses completeness near the Galactic plane, so the authors of the catalog excluded regions with $|b|<5^{\circ}$ for $30^{\circ} \leqslant l \leqslant 330^{\circ}$ and $|b|<8^{\circ}$ otherwise. The SB-58M catalog consists of objects that were detected with a significance greater than $4.8 \sigma$ in the energy range of $14-195 \mathrm{keV}$ in the first 58 months of observation by the Swift BAT. We select the extragalactic objects in this catalog for this work. The catalog of SB-AGN contains AGNs with at least $5 \sigma$ significance in the energy range of $15-55 \mathrm{keV}$ in the first 60 months of observation by the Swift BAT. The 2LAC (Ackermann et al. 2011) data set consists of AGNs detected with at least $4 \sigma$ significance in the energy range of $100 \mathrm{MeV}-100 \mathrm{GeV}$ in the first 24 months of observation by the Fermi-LAT. The Galactic disk region with $|b|<10^{\circ}$ is cut away. We also examine the VCV catalog, which is a compilation of several AGN surveys. The number of objects and the SD events after the cuts are applied in each case are given in Table 2.

\subsection{Methods}

For a given set of parameters $\left(E_{\min }, \psi, z_{\max }\right)$, there are $N$ events with energies $E \geqslant E_{\min }$. We can count the number of events, $k$, out of $N$ that are correlated with objects in a catalog with redshifts $z \leqslant z_{\max }$ and within the angular separation $\psi$. We can calculate the probability, $P$, that $k$ or more correlated events are found from an isotropic UHECR flux under the same conditions. We carry out a parameter scan in $\left(E_{\min }, \psi, z_{\max }\right)$ space to find the set of parameters that maximizes the correlation between the TA events and the catalog objects, i.e., minimizes $P$.
Table 3

Scanned Regions and Step Sizes for Each Scan Parameter

\begin{tabular}{lcc}
\hline \hline Parameter & Range & Step Size \\
\hline Energy (EeV) & $E \geqslant 40$ & Energy of each event by sorted order \\
Redshift $(z)$ & $0.001 \leqslant z \leqslant 0.030$ & 0.001 \\
Window (deg) & $1 \leqslant \psi<8$ & 0.1 \\
& $8 \leqslant \psi \leqslant 15$ & 1 \\
\hline
\end{tabular}

To determine the probability, $P$, we first obtain the probability, $p$, that a random event is correlated with at least one object by chance for a given $\left(\psi, z_{\max }\right)$. To do so, we generate $10^{4}$ randomly distributed events in the same experimental region of each of the catalogs as described in Table 2 and cross-correlate them with the objects.

Then $P$ can be obtained as a cumulative binomial probability:

$$
P=\sum_{j=k}^{N} C_{j}^{N} p^{j}(1-p)^{N-j} .
$$

The scan over parameters is performed as follows. The value of $E_{\min }$ is set by the energy of the $N$ th highest energy event. We scan over all values of $N$ such that $E_{\min }$ is greater than $40 \mathrm{EeV}$. Note that this energy is less than the energy $(50 \mathrm{EeV})$ at which the TA energy spectrum begins to fall off steeply (Abu-Zayyad et al. 2013). We set the upper boundary of the parameter $z_{\max }$ as 0.03 , which corresponds to the distances smaller than $120 \mathrm{Mpc}$. This is comparable to the GZK horizon: at $E>40 \mathrm{EeV}$ about $40 \%$ of the UHECR flux is collected from within this distance (Koers \& Tinyakov 2009). Although choosing a larger maximum distance would increase this fraction, note that the UHECR deflections also increase with distance, as well as the penalty for scanning. The selected step size of $z_{\max }$ is 0.001 , which is the typical accuracy in the redshift measurements. The separation angle, $\psi$, is varied from $1^{\circ}$ to $15^{\circ}$. The maximum search window of $\psi=15^{\circ}$ is selected as appropriate for lower energy events $(\sim 40 \mathrm{EeV})$ arriving from the distance of $100 \mathrm{Mpc}$. The selected step size in $\psi$ is chosen as 0.1 for $\psi<8^{\circ}$ and $1^{\circ}$ for $8^{\circ} \leqslant \psi \leqslant 15^{\circ}$. The parameter ranges and step sizes are summarized in Table 3.

The minimum $P$ obtained from this procedure does not represent the correlation probability directly, because the parameter scanning enhances the correlation probability artificially (Tinyakov \& Tkachev 2004). Therefore, a penalty for parameter scanning (PPS) should be evaluated and the true probability of correlation must include this penalty. This will be described in Section 4.

\section{RESULTS}

\subsection{Results of the Parameter Scan Analysis}

The results of the parameter scan are listed in Table 4 . The smallest value of $P_{\min }^{\text {obs }}$ among all the catalogs is $1.3 \times$ $10^{-5}$ found in the SB-AGN catalog with the best parameters $\left(E_{\min }, \psi, z_{\max }\right)_{\text {best }}=\left(62.20 \mathrm{EeV}, 10^{\circ}, 0.020\right)$. A sky map of the TA events and the objects under the condition $\left(E_{\min }, \psi, z_{\max }\right)_{\text {best }}$ that gives the smallest $P_{\min }^{\text {obs }}$ is shown in Figure 2. All the observed UHECRs with $E \geqslant E_{\min }$ correlate with at least one object with $z \leqslant z_{\max }$ in the SB-AGN catalog. Figures 3 and 4 show the probability as a function of each parameter $\left(E_{\min }, \psi, z_{\max }\right)_{\text {best }}$, while fixing the values of the other two at the optimum value for this data comparison. 

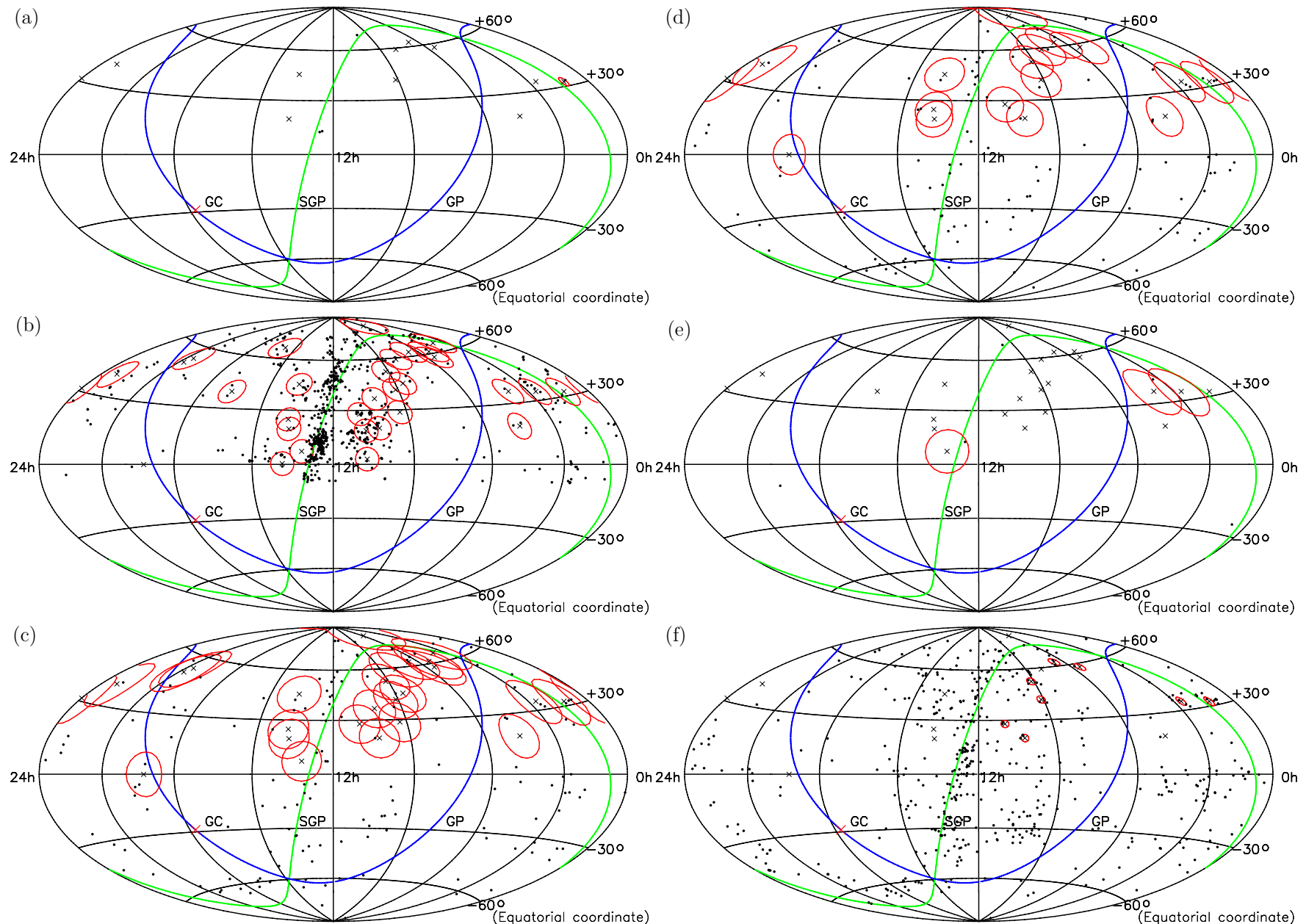

Figure 2. Arrival directions of observed UHECRs with the objects of each of the catalogs [(a) 3CRR, (b) 2MRS, (c) SB-58M, (d) SB-AGN, (e) 2LAC, and (f) VCV]. Dots: catalog objects; $\times$ : arrival direction of observed cosmic rays; circle: window around cosmic ray events, GC: Galactic center, GP: Galactic plane, SGP: supergalactic plane.

(A color version of this figure is available in the online journal.)

Table 4

Summary of Correlations with the Best Parameter Set (Minimum Threshold, Window Size, Maximum Redshift) for Each Catalog

\begin{tabular}{lcccrccccc}
\hline \hline Catalog & $\begin{array}{c}E_{\min } \\
(\mathrm{EeV})\end{array}$ & $\begin{array}{c}\psi \\
(\mathrm{deg})\end{array}$ & $\begin{array}{c}z_{\max } \\
(z)\end{array}$ & $A$ & $N$ & $k$ & $p$ & $P_{\min }$ & $P_{\text {PPS }}$ \\
\hline 3CRR & 66.77 & 2.0 & 0.017 & 4 & 11 & 1 & 0.0020 & $2.2 \times 10^{-2}$ & 0.75 \\
2MRS & 51.85 & 6.5 & 0.005 & 660 & 31 & 29 & 0.62 & $8.5 \times 10^{-5}$ & 0.21 \\
SB-58M & 57.46 & 11 & 0.017 & 79 & 25 & 25 & 0.68 & $6.1 \times 10^{-5}$ & 0.04 \\
SB-AGN & 62.20 & 10 & 0.020 & 58 & 17 & 17 & 0.52 & $1.3 \times 10^{-5}$ & 0.01 \\
2LAC & 55.41 & 12 & 0.018 & 3 & 23 & 3 & 0.069 & $2.1 \times 10^{-1}$ & 0.83 \\
VCV & 62.20 & 2.1 & 0.016 & 288 & 17 & 8 & 0.14 & $8.6 \times 10^{-4}$ & 0.25 \\
\hline
\end{tabular}

Notes. $A$ : number of objects with the redshift $\leqslant z_{\max }, N$ : number of observed cosmic ray events with the energy $E \geqslant E_{\min }, k$ : number of events correlated with objects, $p$ : probability of correlation for a single event from an isotropic distribution, $P_{\min }$ : the cumulative binomial probability to obtain $k$ or more estimated from an isotropic distribution, and $P_{\mathrm{PPS}}$ : the probability after including the penalties from parameter scanning.

Now let us consider the PPS. We evaluate the probability, $P_{\text {PPS }}$, of finding a correlation by chance with $P_{\min }^{\text {sim }}$ smaller than that obtained from the data as follows (for a more detailed description of the penalty calculation see, e.g., Tinyakov \& Tkachev 2004). We generate $10^{4}$ random sets of $N$ "cosmic ray events," where $N$ is the same as the number of the observed events with energies greater than $40 \mathrm{EeV}$. For each of the mock event sets, the parameter scanning is carried out using exactly the same method as for the observed data set, and $P_{\min }^{\operatorname{sim}}$ was calculated. Note that the parameters $\left(E_{\min }, \psi, z_{\max }\right)_{\text {best }}$ that yield $P_{\min }^{\operatorname{sim}}$ are different for each of the $10^{4}$ trials. The distribution of $P_{\min }^{\operatorname{sim}}$ in the case of the SB-AGN catalog is shown in Figure 5 together with $P_{\min }^{\mathrm{obs}}$. One can see that rather small values $P_{\min } \leqslant 1.3 \times 10^{-5}$ can happen even though the simulated UHECR distribution is isotropic.

If we repeat the same experiment and the parameter scanning many times, the value of our result $P_{\mathrm{min}}^{\mathrm{obs}}=1.3 \times 10^{-5}$ could be just a chance occurrence. The probability including the PPS is evaluated as $P_{\mathrm{PPS}}=0.01$ for the SB-AGN catalog, and the values for all the catalogs are listed in Table 4 . The smallest value of $P_{\text {PPS }}$ among the catalogs is 0.01 from the SB-AGN catalog. This does not yet include the penalty for searching in several catalogs.

If we have several catalogs, regardless of whether they are independent or partially overlapping, there is a possibility of finding a catalog that gives the same or smaller $P_{\min }^{\text {obs }}$ value by chance, even though there are no correlations between the events and the objects. The straightforward way to calculate the penalty factor associated with the partially overlapping catalogs, as is the case in our analysis, is to include all the catalogs in the Monte 

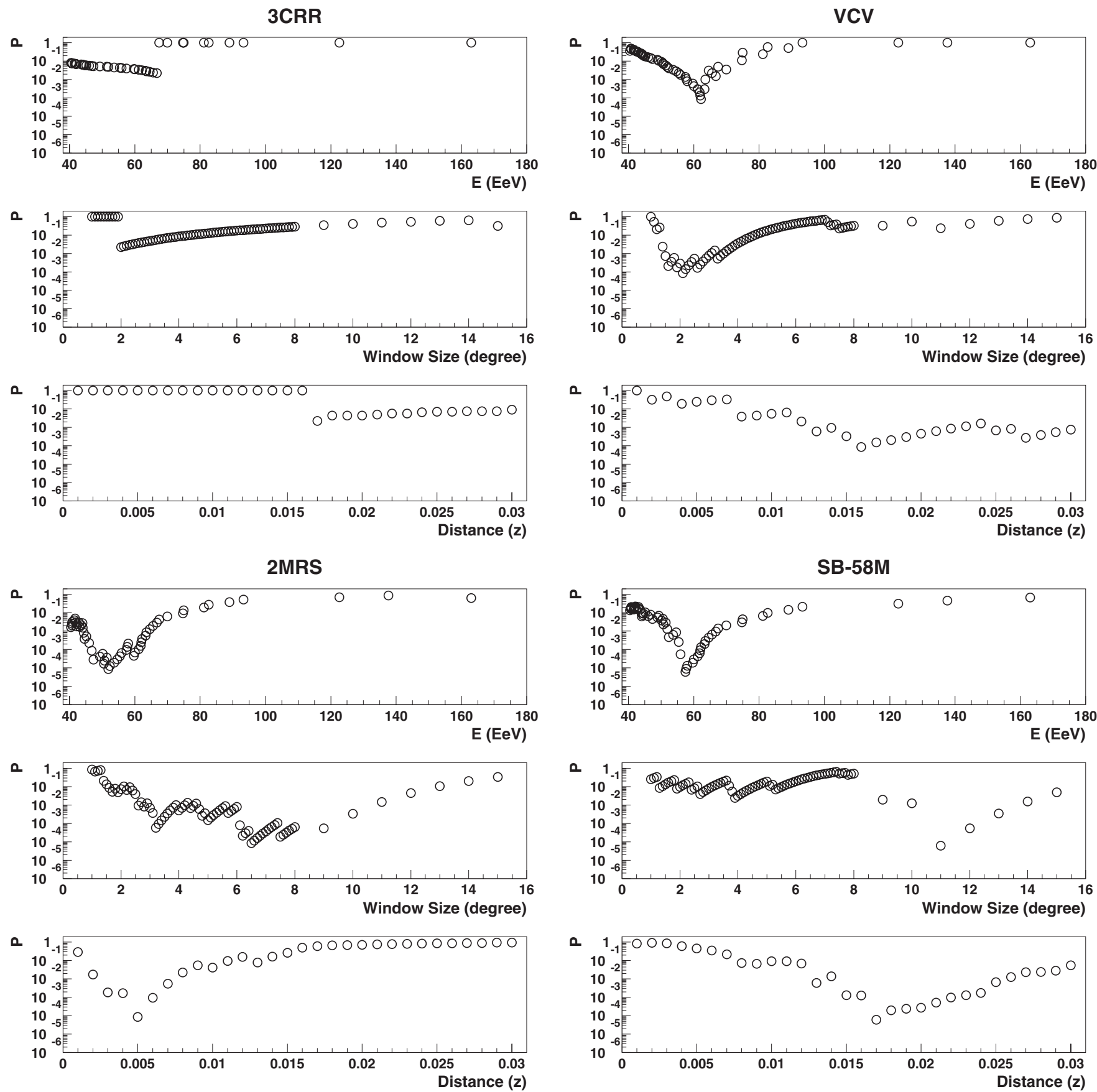

Figure 3. Cumulative binomial probability distribution for the 3CRR (top left panel), VCV (top right panel), 2MRS (bottom left panel), and SB-58M (bottom right panel) catalogs. Each panel shows the probability distribution with the energy threshold $\left(E_{\min }\right)$ of the observed cosmic rays (top), the window $\psi$ (middle), and the redshift $z_{\max }$ (bottom). In each of the plots, the other two parameters are fixed at the parameter set that provides $P_{\min }$.

Carlo simulation. Therefore, we have repeated the simulation with $10^{4}$ mock sets as described above but with the scanning performed in all six catalogs. Calculating the fraction of mock sets that show equal or better correlation than the data, we find the final probability with a PPS and a PCS, $P_{\text {PPS }+ \text { PCS }}=0.09$. Therefore, we conclude that no significant correlation between UHECRs and the astronomical objects is found in the current TA data set.

\subsection{Uncertainties}

First, we consider the effect of finite resolution in the scanning parameters. The uncertainty in determination of the arrival directions and energy only makes correlations worse because of direction smearing and the contamination of energy events lower than $E_{\min }$. Therefore, the obtained $P_{\min }^{\text {obs }}$ already includes these resolution effects. This also concerns the uncertainty in the redshifts of the catalog objects.

Consider the effect of the systematic uncertainty in energy determination. As mentioned above, this uncertainty is $22 \%$ (Abu-Zayyad et al. 2011). Note, however, that the present analysis with the parameter scanning is independent of the absolute energy scale: the energies of the events are no more than keys for event sorting, and a systematic energy shift does not affect the scanning in $E_{\min }$, hence the number of events involved in the correlation with the objects and the probability $P_{\text {min }}^{\text {obs }}$. 

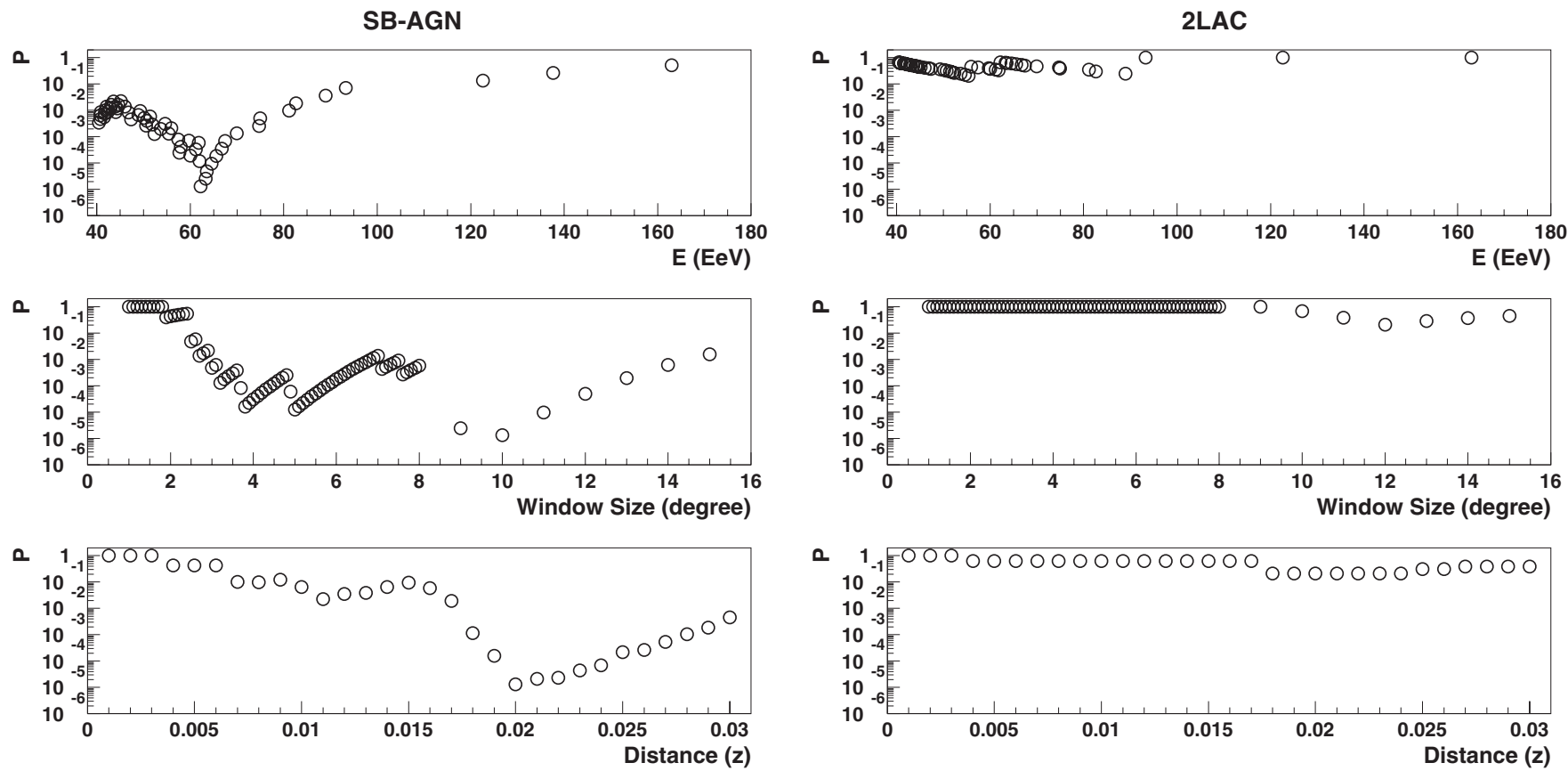

Figure 4. Cumulative binomial probability distribution for the SB-AGN (left panel) and 2LAC (right panel). Each panel shows the probability distribution with the energy threshold $\left(E_{\min }\right)$ of the observed cosmic rays (top), the window $\psi$ (middle), and the redshift $z_{\max }$ (bottom). In each of the plots, the other two parameters are fixed at the optimum value.

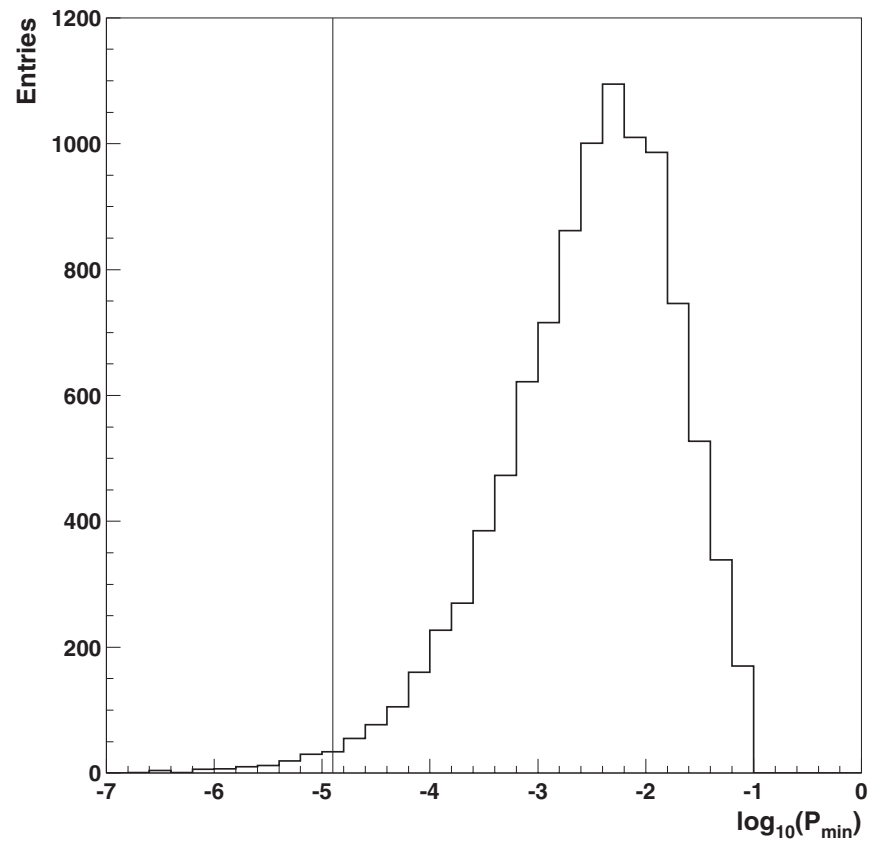

Figure 5. Distribution of the probability $P_{\min }^{\operatorname{sim}}$ for the SB-AGN catalog determined from $10^{4}$ simulated isotropic data sets. The observed $P_{\min }^{\text {obs }}=$ $1.3 \times 10^{-5}$ is shown as a vertical line.

The last issue to discuss is the incompleteness of the catalogs, which remains even after we cut out the regions around the Galactic plane. The objects in the VCV catalog are inhomogeneous because it is a mere compilation of objects detected under different conditions. The completeness of the other catalogs, in particular the $2 \mathrm{LAC}$, could be affected by our cuts, particularly by the selection of objects with the known redshift. While the incompleteness may make the interpretation of correlations ambiguous if they are present, it does not affect the calculation of $P_{\mathrm{min}}^{\mathrm{obs}}$. In fact, the effect of the incompleteness cancels out in $P_{\mathrm{min}}^{\text {obs }}$ since the same set of objects is used to cross-correlate with the data and each mock event set. Therefore, the incompleteness of the catalogs cannot produce spurious correlations (although it may, in principle, be responsible for their absence).

\section{DISCUSSION}

\subsection{Search for Correlations with a Specific Type of Object}

So far we have treated the objects in each catalog equally, regardless of their class. Now let us examine whether there is a specific type of object that has stronger correlations with UHECRs than others. We will consider the case of the SB-AGN catalog that shows strongest correlations with UHECRs.

First, we count the number of objects of each class in the TA FOV with redshifts smaller than 0.02. Some of the objects are labeled "unclassified" in the catalog. For these we used the information from other surveys (Noguchi et al. 2010; Parisi 2011; Veron-Cetty \& Veron 2010; Tueller et al. 2010). The fractions of Seyfert 2, 1, 1.5, 1.9, and LINER galaxies in the SB-AGN catalog satisfying the above conditions are 0.441 , $0.235,0.132,0.044$, and 0.044 , respectively (the total fraction of other class AGNs: 0.044; the fraction of the unclassified AGN: 0.059). The total number of AGNs that are correlated with UHECRs is 22 with the parameters in Table 4 (note that the number of UHECR events and that of AGNs are not the same because some of the events fall within the given angular distance from several sources). Among these 22 AGNs, the fractions of Seyfert 2, 1, 1.5, 1.9, LINER, and unclassified galaxies are $0.455,0.182,0.227,0.045,0.045$, and 0.045 , respectively. We see that the largest difference is for the Seyfert 1.5 galaxies. The probability, $P$, of finding 5 or more correlated Seyfert 1.5 galaxies out of 22 can be evaluated by the cumulative binomial 
probability with an expectation of 0.13 and is $P=0.16$. Therefore, no significant correlation with a specific type of AGN in the SB-AGN catalog is found.

\section{CONCLUSION}

We examine the correlations between the observed UHECR arrival directions and the extragalactic objects from the different survey catalogs under the assumption that the sources have the same intrinsic UHECR luminosities. We use the TA-SD events with energies greater than $40 \mathrm{EeV}$ obtained in the first 40 months of observation. We search for maximum correlations by scanning over three parameters $E_{\min }, \psi$, and $z_{\max }$ in six different catalogs. The smallest probability among these six catalogs was found with the Swift BAT (60 month) AGN catalog, $P_{\text {min }}^{\text {obs }}=1.3 \times 10^{-5}$. This probability increases to $P_{\text {PPS }}=0.01$ when we include the penalty for the three-parameter scanning in the Swift BAT catalog alone and to $P_{\mathrm{PPS}+\mathrm{PCS}}=0.09$ when scanning in all the catalogs is taken into account. Therefore, we conclude that no significant correlation with the considered catalogs of extragalactic objects is found in the present TA data set. Investigating specifically the case of the Swift BAT (60 month) AGN catalog that gives the strongest correlation, we find that no particular subclass of objects is responsible for this correlation.

The TA experiment is supported by the Japan Society for the Promotion of Science through Grants-in-Aid for Scientific Research on Specially Promoted Research (21000002) "Extreme Phenomena in the Universe Explored by Highest Energy Cosmic Rays" and the Inter-University Research Program of the Institute for Cosmic Ray Research; by the US National Science Foundation awards PHY-0307098, PHY-0601915, PHY0703893, PHY-0758342, PHY-0848320, PHY-1069280, PHY1069286 (Utah), and PHY-0649681 (Rutgers); by the National Research Foundation of Korea (2006-0050031, 2007-0056005, 2007-0093860, 2010-0028071, 2013-004883, R32-10130); and by the Russian Academy of Sciences, RFBR grants 10-0201406a and 11-02-01528a (INR), IISN project No. 4.4509.10, and Belgian Science Policy under IUAP VI/11 (ULB). The foundations of Dr. Ezekiel R. and Edna Wattis Dumke, Willard L. Eccles, and George S. and Dolores Dore Eccles all helped with generous donations. The state of Utah supported the project through its Economic Development Board, and the University of Utah through the Office of the Vice President for Research. The experimental site became available through the cooperation of the Utah School and Institutional Trust Lands Administration (SITLA), the US Bureau of Land Management, and the US Air Force. We also wish to thank the people and the officials of Millard County, Utah, for their steadfast and warm support. We gratefully acknowledge the contributions from the technical staffs of our home institutions as well as the University of Utah Center for High Performance Computing (CHPC).

\section{REFERENCES}

Abbasi, R. U., Abu-Zayyad, T., Allen, M., et al. 2008a, APh, 30, 175 Abbasi, R. U., Abu-Zayyad, T., Allen, M., et al. 2008b, PhRvL, 100, 101101 Abraham, J., Abreu, P., Aglietta, M., et al. 2008, PhRvL, 101, 061101

Abraham, J., Abreu, P., Aglietta, M., et al. 2010, PhLB, 685, 239

Abreu, P., Aglietta, M., Aguirre, C., et al. 2007, Sci, 318, 938

Abreu, P., Aglietta, M., Aguirre, C., et al. 2008, APh, 29, 188

Abreu, P., Aglietta, M., Ahn, E. J., et al. 2010, APh, 34, 314

Abu-Zayyad, T., Aida, R., Allen, M., et al. 2012a, ApJ, 757, 26

Abu-Zayyad, T., Aida, R., Allen, M., et al. 2012b, NIMPA, 689, 87

Abu-Zayyad, T., Aida, R., Allen, M., et al. 2012c, APh, 39, 109

Abu-Zayyad, T., Aida, R., Allen, M., et al. 2013, ApJL, 768, L1

Abu-Zayyad, T., Allen, M., Barcikowski, E., et al. 2011, Proceedings of 32nd International Cosmic Ray Conference, 2, 250

Ackermann, M., Ajello, M., Allafort, A., et al. 2011, ApJ, 743, 171

Ajello, M., Alexander, D. M., Greiner, J., et al. 2012, ApJ, 749, 21

Greisen, K. 1966, PhRvL, 16, 748

Han, J. L., Manchester, R. N., Lyne, A. G., Qiao, G. J., \& van Straten, W. 2006, ApJ, 642, 868

Huchra, J. P., Macri, L. M., Masters, K. L., et al. 2012, ApJS, 199, 26

Ivanov, D., Stokes, B. P., Thompson, G. B., et al. 2011, Proc. of 32nd International Cosmic Ray Conference, 2, 258

Kawai, H., Yoshida, S., Yoshii, H., et al. 2008, NuPhS, 175, 221

Koers, H. B. J., \& Tinyakov, P. 2009, JCAP, 04, 003

Kotera, K., \& Olinto, A. V. 2011, ARA\&A, 49, 119

Kronberg, P. P. 1994, RPPh, 57, 325

Laing, R. A., Riley, J. M., \& Longair, M. S. 1983, MNRAS, 204, 151 (data taken from http://3crr.extragalactic.info)

Noguchi, K., Terashima, Y., Ishino, Y., et al. 2010, ApJ, 711, 144

Parisi, P. 2011, arXiv:1105.0527

Pshirkov, M., Tinyakov, P., Kronberg, P., \& Newton-McGee, K. 2011, ApJ, 738,192

Sun, X. H., Reich, W., Waelkens, A., \& Enbßlin, T. A. 2008, A\&A, 477, 573

Tokuno, H., Tameda, Y., Takeda, M., et al. 2012, NIMPA, 676, 54

Tinyakov, P. G., \& Tkachev, I. I. 2004, PhRvD, 69, 128301

Tueller, J., Baumgartner, W. H., Markwardt, C. B., et al. 2010, ApJS, 186 , 378 (the latest data taken from http://heasarc.nasa.gov/docs/swift/results/ bs $58 \mathrm{mon} /$ )

Veron-Cetty, M. P., \& Veron, P. 2006, A\&A , 455, 773

Veron-Cetty, M. P., \& Veron, P. 2010, A\&A, 518, A10

Zatsepin, G. T., \& Kuz'min, V. A. 1966, JETPL, 4, 78 